\title{
Random fixed point theorem on a Ćirić-type contractive mapping and its consequence
}

\author{
M Saha ${ }^{1}$ and Anamika Ganguly²
}

"Correspondence: anamika.simi@gmail.com

${ }^{2}$ Burdwan Railway Balika Vidyapith High School, Khalasipara, Burdwan, West Bengal 713101, India Full list of author information is available at the end of the article

\begin{abstract}
Purpose: The main purpose of this paper is to prove a random fixed point theorem in a separable Banach space equipped with a complete probability measure for a certain class of contractive mappings.

Results: The main finding of this paper is the identification of some random fixed point theorems and the relevant application with appropriate supporting examples.

Conclusion: A random fixed point theorem is useful to determine the existence of a solution in a Banach space of a random nonlinear integral equation.

MSC: Primary $47 \mathrm{H} 10$; secondary $60 \mathrm{H} 25$

Keywords: complete probability measure space; random variable; random solution; random fixed point equation; Bochner integral
\end{abstract}

\section{Introduction}

The application of fixed point theory in different branches of mathematics, statistics, engineering and economics relating to problems associated with approximation theory, theory of differential equations, theory of integral equations, etc. has been recognized in the existing literature [1,2] and [3]. Progress in the study on fixed points of non-expansive mappings, contractive mappings in various spaces like a metric space, a Banach space, a fuzzy metric space, a cone metric space $e t c$. has been saturated at large. After the initial impetus given by the Prague school of Probability in 1950s, considerable attention has been given to the study of random fixed point theorems. This arises because of the significance of fixed point theorems in probabilistic functional analysis and probabilistic models along with several applications. Issues relating to measurability of solutions, probabilistic and statistical aspects of random solutions have arisen due to the introduction of randomness. It is no denying the fact that random fixed point theorems are stochastic generalizations of classical fixed point theorems that have been described as deterministic results.

Špaček [4] and Hanš [5, 6] first proved random fixed point theorems for random contraction mappings on separable complete metric spaces. The article by Bharucha-Reid [7] in 1976 attracted the attention of several mathematicians and led to the development of this theory. Špaček's and Hanšss theorems have been extended to multivalued contraction mappings by Itoh [8]. A random version of Schaduer's fixed point theorem on an atomic probability measure space has been provided by Mukherjee [9]. The results of this work have been generalized by Bharucha-Reid $[1,7]$ on a general probability measure space. Itoh [8] obtained random fixed point theorems with an application to random differential 
equations in Banach spaces. Several random fixed point theorems including random analogue of the classical results based on Rothe [10] have been obtained by Sehgal and Waters [11]. Kumam in a series of papers (see [12-25]) proved some remarkable results on random fixed point theorems. In a couple of papers [17] and [19], he along with his coauthor proved some random fixed point theorems for multivalued non-expansive non-self operators in the framework of Banach spaces satisfying inwardness conditions. In another paper, $\mathrm{Ku}$ mam and Plubtieng [13] proved some random coincidence points and random common fixed point theorems for nonlinear multivalued random operators. They also proved the existence of a random coincidence point for a pair of reciprocally continuous and compatible single-valued and multivalued operators. Saha [26], Saha and Debnath [27] in their works established some random fixed point theorems over a separable Banach space and a separable Hilbert space. On the other hand, Padgett [28] applied a random fixed point theorem to prove the existence of a solution in a Banach space of a random nonlinear integral equation. Achari [29], Saha and Dey [30] developed this new area of application.

Banach's contraction principle [31] is one of the pivotal results of nonlinear analysis. It has been the source of metric fixed point theory and its significance rests in its vast applicability in different branches of mathematics. In the general setting of a complete metric space, this theorem runs as follows (see Theorem 2.1 [32] or Theorem 1.2.2 [33]).

Theorem 1.1 (Banach's contraction principle) Let $(X, d)$ be a complete metric space, $c \in$ $[0,1)$ and $f: X \rightarrow X$ be a mapping such that for each $x, y \in X, d(f x, f y) \leq c d(x, y)$.

Then $f$ has a unique fixed point $a \in X$, and for each $x \in X, \lim _{n \rightarrow \infty} f^{n} x=a$.

On the other hand, Greguš [34] proved the following fixed point theorem.

Theorem 1.2 Let $X$ be a Banach space, $C$ be a closed convex subset of $X$ and $T: C \rightarrow C$ be a mapping satisfying

$$
\|T x-T y\| \leq a\|x-y\|+p\|T x-x\|+p\|T y-y\|
$$

for all $x, y \in C$, where $0<a<1, p \geq 0$ and $a+2 p=1$. Then $T$ has a unique fixed point.

During the eighties, many theorems which are closely related to the Greguš theorem have appeared in several literatures (see [35-38] and [39]). Also, Cirić [40] dealt with a class of mappings (not necessarily continuous) which are defined on a metric space and proved the following fixed point theorem which is a double generalization of Greguš [34].

Theorem 1.3 Let $C$ be a closed convex subset of a complete convex metric space $X$ and $T: C \rightarrow C$ be a mapping satisfying

$$
\begin{aligned}
d(T x, T y) \leq & a \max \{d(x, y), c[d(x, T y)+d(y, T x)]\} \\
& +b \max \{d(x, T x), d(y, T y)\}
\end{aligned}
$$

where $0<a<1, a+b=1, c \leq \frac{4-a}{8-a}$ for all $x, y \in C$. Then $T$ has a unique fixed point.

In light of Theorem 1.3, the following theorem has been proved by Ćirić [40]. 
Theorem 1.4 Let $C$ be a closed convex metric space $X$ and $T: C \rightarrow C$ be a mapping satisfying

$$
\begin{aligned}
d(T x, T y) \leq & a d(x, y)+b \max \{d(x, T x), d(y, T y)\} \\
& +c[d(x, T y)+d(y, T x)]
\end{aligned}
$$

for all $x, y \in C$, where $0 \leq a<1, b \geq 0, c \geq 0, a+c>0$ and $a+b+\frac{7}{3} c=1$. Then $T$ has $a$ unique fixed point.

Ćirić also introduced several contractive operators on metric spaces and proved many fixed point theorems on such operators. Inspired by Ćirić's contractive operators, many researchers have obtained fixed point theorems on Ćirićs operators in different settings. In this context, Karapinar [41] proved some non-unique fixed point theorems on Ćirićtype contractive operators in cone metric spaces. Also Karapinar et al. [42] proved a fixed point theorem on a metric space for a class of maps that satisfy the Ćirić-type contractive condition.

In this paper, our main objective is to prove some random fixed point theorems in a separable Banach space equipped with a complete probability measure for a certain class of contractive mappings. The results are stochastic generalizations of deterministic fixed point theorems of Ćirić [40]. The result obtained in this paper will also be useful in application to a random nonlinear integral equation. Also, we have introduced some appropriate supporting examples.

In order to make the paper self-contained, we state some important definitions and an example that are available in Joshi and Bose [3] and Debnath and Mikusinski [2].

\section{Preliminaries}

Let $\left(X, \beta_{X}\right)$ be a separable Banach space, where $\beta_{X}$ is a $\sigma$-algebra of Borel subsets of $X$, and let $(\Omega, \beta, \mu)$ denote a complete probability measure space with measure $\mu$ and $\beta$ be a $\sigma$-algebra of subsets of $\Omega$. For more details, one can see Joshi and Bose [3].

Definition 2.1 A mapping $x: \Omega \rightarrow X$ is said to be an $X$-valued random variable if the inverse image under the mapping $x$ of every Borel set $B$ of $X$ belongs to $\beta$, that is, $x^{-1}(B) \in \beta$ for all $B \in \beta_{X}$.

Definition 2.2 A mapping $x: \Omega \rightarrow X$ is said to be a finitely-valued random variable if it is constant on each finite number of disjoint sets $A_{i} \in \beta$ and is equal to 0 on $\Omega-\left(\bigcup_{i=1}^{n} A_{i}\right)$. $x$ is called a simple random variable if it is finitely valued and $\mu\{\omega:\|x(\omega)\|>0\}<\infty$.

Definition 2.3 A mapping $x: \Omega \rightarrow X$ is said to be a strong random variable if there exists a sequence $\left\{x_{n}(\omega)\right.$ \} of simple random variables which converges to $x(\omega)$ almost surely, that is, there exists a set $A_{0} \in \beta$ with $\mu\left(A_{0}\right)=0$ such that $\lim _{n \rightarrow \infty} x_{n}(\omega)=x(\omega), \omega \in \Omega-A_{0}$.

Definition 2.4 A mapping $x: \Omega \rightarrow X$ is said to be a weak random variable if the function $x^{\prime \prime}(x(\omega))$ is a real-valued random variable for each $x^{\prime \prime} \in X^{*}$, the space $X^{\prime \prime}$ denoting the first normed dual space of $X$. 
In a separable Banach space $X$, the notions of strong and weak random variables $x: \Omega \rightarrow$ $X$ (see Corollary 1 of Joshi and Bose [3]) coincide, and in respect of such a space $X, x$ is termed as a random variable.

We recall the following results.

Theorem 2.5 (see Theorem 6.1.2(a) of Joshi and Bose [3]) Let x, $y: \Omega \rightarrow X$ be strong random variables and $\alpha, \beta$ be constants. Then the following statements hold:

(a) $\alpha x(\omega)+\beta y(\omega)$ is a strong random variable.

(b) If $f(\omega)$ is a real-valued random variable and $x(\omega)$ is a strong random variable, then $f(\omega) x(\omega)$ is a strong random variable.

(c) If $\left\{x_{n}(\omega)\right\}$ is a sequence of strong random variables converging strongly to $x(\omega)$ almost surely, i.e., if there exists a set $A_{0} \in \beta$ with $\mu\left(A_{0}\right)=0$ such that $\lim _{n \rightarrow \infty}\left\|x_{n}(\omega)-x(\omega)\right\|=0$ for every $\omega \notin A_{0}$, then $x(\omega)$ is a strong random variable.

Remark 2.6 If $X$ is a separable Banach space, then every strong and also weak random variable is measurable in the sense of Definition 2.1.

Let $Y$ be another Banach space. We also need the following definitions as cited in Joshi and Bose [3].

Definition 2.7 A mapping $F: \Omega \times X \rightarrow Y$ is said to be a random mapping if $F(\omega, x)=Y(\omega)$ is a $Y$-valued random variable for every $x \in X$.

Definition 2.8 A mapping $F: \Omega \times X \rightarrow Y$ is said to be a continuous random mapping if the set of all $\omega \in \Omega$ for which $F(\omega, x)$ is a continuous function of $x$ has measure one.

Definition 2.9 A mapping $F: \Omega \times X \rightarrow Y$ is said to be demi-continuous at the $x \in X$ if $\left\|x_{n}-x\right\| \rightarrow 0$ implies $F\left(\omega, x_{n}\right) \stackrel{\text { weakly }}{\longrightarrow} F(\omega, x)$ almost surely.

Theorem 2.10 (see Theorem 6.2.2 of Joshi and Bose [3]) Let $F: \Omega \times X \rightarrow Y$ be a demicontinuous random mapping where $a$ Banach space $Y$ is separable. Then, for any $X$-valued random variable $x$, the function $F(\omega, x(\omega))$ is a $Y$-valued random variable.

Remark 2.11 (see [3]) Since a continuous random mapping is a demi-continuous random mapping, Theorem 2.5 is also true for a continuous random mapping.

We shall also recall the following definitions as seen in Joshi and Bose [3].

Definition 2.12 An equation of the type $F(\omega, x(\omega))=x(\omega)$, where $F: \Omega \times X \rightarrow X$ is a random mapping, is called a random fixed point equation.

Definition 2.13 Any mapping $x: \Omega \rightarrow X$ which satisfies the random fixed point equation $F(\omega, x(\omega))=x(\omega)$ almost surely is said to be a wide sense solution of the fixed point equation.

Definition 2.14 Any $X$-valued random variable $x(\omega)$ which satisfies $\mu\{\omega: F(\omega, x(\omega))=$ $x(\omega)\}=1$ is said to be a random solution of the fixed point equation or a random fixed point of $F$. 
Remark 2.15 A random solution is a wide sense solution of the fixed point equation. But the converse is not necessarily true. This is evident from the following example as found under Remark 1 in the work of Joshi and Bose [3].

Example 2.16 Let $X$ be the set of all real numbers and let $E$ be a non-measurable subset of $X$. Let $F: \Omega \times X \rightarrow Y$ be a random mapping defined as $F(\omega, x)=x^{2}+x-1$ for all $\omega \in \Omega$.

In this case, the real-valued function $x(\omega)$, defined as $x(\omega)=1$ for all $\omega \in \Omega$, is a random fixed point of $F$. However, the real-valued function $y(\omega)$ defined as

$$
y(\omega)= \begin{cases}-1, & \omega \notin E, \\ 1, & \omega \in E\end{cases}
$$

is a wide sense solution of the fixed point equation $F(\omega, x(\omega))=x(\omega)$ without being a random fixed point of $F$.

\section{Main results}

Theorem 3.1 Let $X$ be a separable Banach space and $(\Omega, \beta, \mu)$ be a complete probability measure space. Let $T: \Omega \times X \rightarrow X$ be a continuous random operator such that for $\omega \in \Omega$, T satisfies

$$
\begin{aligned}
\left\|T\left(\omega, x_{1}\right)-T\left(\omega, x_{2}\right)\right\| \leq & a(\omega) \max \left\{\left\|x_{1}-x_{2}\right\|, c(\omega)\left[\left\|x_{1}-T\left(\omega, x_{2}\right)\right\|+\left\|x_{2}-T\left(\omega, x_{1}\right)\right\|\right]\right\} \\
& +b(\omega) \max \left\{\left\|x_{1}-T\left(\omega, x_{1}\right)\right\|,\left\|x_{2}-T\left(\omega, x_{2}\right)\right\|\right\}
\end{aligned}
$$

for all $x_{1}, x_{2} \in X$, where $a(\omega), b(\omega), c(\omega)$ are real-valued random variables such that $0<$ $a(\omega)<1, a(\omega)+b(\omega)=1, c(\omega) \leq \frac{4-a(\omega)}{8-a(\omega)}$ almost surely.

Then there exists a unique random fixed point of $T$ in $X$.

Proof Let $A=\{\omega \in \Omega: T(\omega, x)$ is a continuous function of $x\}$

$$
\begin{aligned}
& B=\{\omega \in \Omega: 0<a(\omega)<1\} \cap\{\omega \in \Omega: a(\omega)+b(\omega)=1\} \\
& \cap\left\{\omega \in \Omega: c(\omega) \leq \frac{4-a(\omega)}{8-a(\omega)}\right\} \\
& C_{x_{1}, x_{2}}=\left\{\omega \in \Omega:\left\|T\left(\omega, x_{1}\right)-T\left(\omega, x_{2}\right)\right\|\right. \\
& \quad \leq a(\omega) \max \left\{\left\|x_{1}-x_{2}\right\|, c(\omega)\left[\left\|x_{1}-T\left(\omega, x_{2}\right)\right\|+\left\|x_{2}-T\left(\omega, x_{1}\right)\right\|\right]\right\} \\
& \left.\quad+b(\omega) \max \left\{\left\|x_{1}-T\left(\omega, x_{1}\right)\right\|,\left\|x_{2}-T\left(\omega, x_{2}\right)\right\|\right\}\right\} .
\end{aligned}
$$

Let $S$ be a countable dense subset of $X$. We now prove that

$$
\bigcap_{x_{1}, x_{2} \in X}\left(C_{x_{1}, x_{2}} \cap A \cap B\right)=\bigcap_{s_{1}, s_{2} \in S}\left(C_{s_{1}, s_{2}} \cap A \cap B\right) .
$$

Then for all $s_{1}, s_{2} \in S$,

$$
\begin{aligned}
\left\|T\left(\omega, s_{1}\right)-T\left(\omega, s_{2}\right)\right\| \leq & a(\omega) \max \left\{\left\|s_{1}-s_{2}\right\|, c(\omega)\left[\left\|s_{1}-T\left(\omega, s_{2}\right)\right\|+\left\|s_{2}-T\left(\omega, s_{1}\right)\right\|\right]\right\} \\
& +b(\omega) \max \left\{\left\|s_{1}-T\left(\omega, s_{1}\right)\right\|,\left\|s_{2}-T\left(\omega, s_{2}\right)\right\|\right\} .
\end{aligned}
$$


Since $S$ is dense in $X$, given $\delta_{i}\left(x_{i}\right)>0(i=1,2)$, there exist $s_{1}, s_{2} \in S$ such that $\left\|x_{i}-s_{i}\right\|<\delta_{i}\left(x_{i}\right)$; $i=1,2$.

Let $x_{1}, x_{2} \in X$.

Note that

$$
\begin{aligned}
& \left\|s_{1}-T\left(\omega, s_{1}\right)\right\| \leq\left\|s_{1}-x_{1}\right\|+\left\|x_{1}-T\left(\omega, x_{1}\right)\right\|+\left\|T\left(\omega, x_{1}\right)-T\left(\omega, s_{1}\right)\right\|, \\
& \left\|s_{1}-s_{2}\right\| \leq\left\|s_{1}-x_{1}\right\|+\left\|x_{1}-x_{2}\right\|+\left\|x_{2}-s_{2}\right\|, \\
& \left\|s_{1}-T\left(\omega, s_{2}\right)\right\| \leq\left\|s_{1}-x_{1}\right\|+\left\|x_{1}-T\left(\omega, x_{2}\right)\right\|+\left\|T\left(\omega, x_{2}\right)-T\left(\omega, s_{2}\right)\right\|, \\
& \left\|s_{2}-T\left(\omega, s_{1}\right)\right\| \leq\left\|s_{2}-x_{2}\right\|+\left\|x_{2}-T\left(\omega, x_{1}\right)\right\|+\left\|T\left(\omega, x_{1}\right)-T\left(\omega, s_{1}\right)\right\|, \\
& \left\|s_{2}-T\left(\omega, s_{2}\right)\right\| \leq\left\|s_{2}-x_{2}\right\|+\left\|x_{2}-T\left(\omega, x_{2}\right)\right\|+\left\|T\left(\omega, x_{2}\right)-T\left(\omega, s_{2}\right)\right\| .
\end{aligned}
$$

We now examine the following cases.

Case I:

Suppose

$$
\left\|T\left(\omega, s_{1}\right)-T\left(\omega, s_{2}\right)\right\| \leq a(\omega)\left\|s_{1}-s_{2}\right\|+b(\omega)\left\|s_{1}-T\left(\omega, s_{1}\right)\right\| .
$$

Now

$$
\begin{aligned}
\left\|T\left(\omega, x_{1}\right)-T\left(\omega, x_{2}\right)\right\| \leq & \left\|T\left(\omega, x_{1}\right)-T\left(\omega, s_{1}\right)\right\|+\left\|T\left(\omega, s_{1}\right)-T\left(\omega, s_{2}\right)\right\| \\
& +\left\|T\left(\omega, s_{2}\right)-T\left(\omega, x_{2}\right)\right\| \\
\leq & \left\|T\left(\omega, x_{1}\right)-T\left(\omega, s_{1}\right)\right\|+\left\|T\left(\omega, s_{2}\right)-T\left(\omega, x_{2}\right)\right\| \\
& +a(\omega)\left\|s_{1}-s_{2}\right\|+b(\omega)\left\|s_{1}-T\left(\omega, s_{1}\right)\right\| .
\end{aligned}
$$

Using (3.2), (3.3), (3.7), we get by routine calculation

$$
\begin{aligned}
\left\|T\left(\omega, x_{1}\right)-T\left(\omega, x_{2}\right)\right\| \leq & (1+b(\omega))\left\|T\left(\omega, x_{1}\right)-T\left(\omega, s_{1}\right)\right\|+\left\|T\left(\omega, s_{2}\right)-T\left(\omega, x_{2}\right)\right\| \\
& +(a(\omega)+b(\omega))\left\|s_{1}-x_{1}\right\|+b(\omega)\left\|x_{1}-T\left(\omega, x_{1}\right)\right\| \\
& +a(\omega)\left\|x_{1}-x_{2}\right\|+a(\omega)\left\|x_{2}-s_{2}\right\| .
\end{aligned}
$$

Since for a particular $\omega \in \Omega, T(\omega, x)$ is a continuous function of $x$, so for any $\varepsilon>0$, there exists $\delta_{i}\left(x_{i}\right)>0(i=1,2)$ such that

$$
\left\|T\left(\omega, x_{1}\right)-T\left(\omega, s_{1}\right)\right\|<\frac{\varepsilon}{8} \quad \text { whenever }\left\|x_{1}-s_{1}\right\|<\delta_{1}\left(x_{1}\right)
$$

and

$$
\left\|T\left(\omega, x_{2}\right)-T\left(\omega, s_{2}\right)\right\|<\frac{\varepsilon}{8} \quad \text { whenever }\left\|x_{2}-s_{2}\right\|<\delta_{2}\left(x_{2}\right) .
$$

Now choose

$$
\delta_{1}=\min \left(\delta_{1}\left(x_{1}\right), \frac{\varepsilon}{8}\right)
$$


and

$$
\delta_{2}=\min \left(\delta_{2}\left(x_{2}\right), \frac{\varepsilon}{8}\right) .
$$

For such a choice of $\delta_{1}, \delta_{2}$ by (3.8), we get

$$
\begin{aligned}
\left\|T\left(\omega, x_{1}\right)-T\left(\omega, x_{2}\right)\right\| \leq & (1+b(\omega)) \frac{\varepsilon}{8}+\frac{\varepsilon}{8}+(a(\omega)+b(\omega)) \frac{\varepsilon}{8} \\
& +a(\omega) \frac{\varepsilon}{8}+b(\omega)\left\|x_{1}-T\left(\omega, x_{1}\right)\right\|+a(\omega)\left\|x_{1}-x_{2}\right\| \\
= & \frac{\varepsilon}{2}+a(\omega)\left\|x_{1}-x_{2}\right\|+b(\omega)\left\|x_{1}-T\left(\omega, x_{1}\right)\right\| .
\end{aligned}
$$

As $\varepsilon>0$ is arbitrary, it follows that

$$
\left\|T\left(\omega, x_{1}\right)-T\left(\omega, x_{2}\right)\right\| \leq a(\omega)\left\|x_{1}-x_{2}\right\|+b(\omega)\left\|x_{1}-T\left(\omega, x_{1}\right)\right\| .
$$

Case II:

Suppose

$$
\begin{aligned}
\left\|T\left(\omega, s_{1}\right)-T\left(\omega, s_{2}\right)\right\| \leq & a(\omega) c(\omega)\left[\left\|s_{1}-T\left(\omega, s_{2}\right)\right\|+\left\|s_{2}-T\left(\omega, s_{1}\right)\right\|\right] \\
& +b(\omega)\left\|s_{1}-T\left(\omega, s_{1}\right)\right\| .
\end{aligned}
$$

Now

$$
\begin{aligned}
\left\|T\left(\omega, x_{1}\right)-T\left(\omega, x_{2}\right)\right\| \leq & \left\|T\left(\omega, x_{1}\right)-T\left(\omega, s_{1}\right)\right\|+\left\|T\left(\omega, s_{1}\right)-T\left(\omega, s_{2}\right)\right\| \\
& +\left\|T\left(\omega, s_{2}\right)-T\left(\omega, x_{2}\right)\right\| \\
\leq & \left\|T\left(\omega, x_{1}\right)-T\left(\omega, s_{1}\right)\right\|+\left\|T\left(\omega, s_{2}\right)-T\left(\omega, x_{2}\right)\right\| \\
& +a(\omega) c(\omega)\left[\left\|s_{1}-T\left(\omega, s_{2}\right)\right\|+\left\|s_{2}-T\left(\omega, s_{1}\right)\right\|\right] \\
& +b(\omega)\left\|s_{1}-T\left(\omega, s_{1}\right)\right\| .
\end{aligned}
$$

Using (3.2), (3.4), (3.5), (3.14), by routine calculation, we get

$$
\begin{aligned}
\left\|T\left(\omega, x_{1}\right)-T\left(\omega, x_{2}\right)\right\| \leq & (1+a(\omega) c(\omega)+b(\omega))\left\|T\left(\omega, x_{1}\right)-T\left(\omega, s_{1}\right)\right\| \\
& +(1+a(\omega) c(\omega))\left\|T\left(\omega, x_{2}\right)-T\left(\omega, s_{2}\right)\right\| \\
& +(a(\omega) c(\omega)+b(\omega))\left\|s_{1}-x_{1}\right\|+a(\omega) c(\omega)\left\|s_{2}-x_{2}\right\| \\
& +a(\omega) c(\omega)\left[\left\|x_{1}-T\left(\omega, x_{2}\right)\right\|+\left\|x_{2}-T\left(\omega, x_{1}\right)\right\|\right] \\
& +b(\omega)\left\|x_{1}-T\left(\omega, x_{1}\right)\right\| .
\end{aligned}
$$

Since for a particular $\omega \in \Omega, T(\omega, x)$ is a continuous function of $x$, by using (3.9), (3.10), (3.11), (3.12) and for such a choice of $\delta_{1}, \delta_{2}$, we get by (3.15)

$$
\begin{aligned}
\left\|T\left(\omega, x_{1}\right)-T\left(\omega, x_{2}\right)\right\| \leq & (1+a(\omega) c(\omega)+b(\omega)) \frac{\varepsilon}{8}+(1+a(\omega) c(\omega)) \frac{\varepsilon}{8} \\
& +(a(\omega) c(\omega)+b(\omega)) \frac{\varepsilon}{8}+a(\omega) c(\omega) \frac{\varepsilon}{8}
\end{aligned}
$$




$$
\begin{aligned}
& +a(\omega) c(\omega)\left[\left\|x_{1}-T\left(\omega, x_{2}\right)\right\|+\left\|x_{2}-T\left(\omega, x_{1}\right)\right\|\right] \\
& +b(\omega)\left\|x_{1}-T\left(\omega, x_{1}\right)\right\| \\
< & \frac{24-4 a(\omega)}{8-a(\omega)} \frac{\varepsilon}{4}+a(\omega) c(\omega)\left[\left\|x_{1}-T\left(\omega, x_{2}\right)\right\|+\left\|x_{2}-T\left(\omega, x_{1}\right)\right\|\right] \\
& +b(\omega)\left\|x_{1}-T\left(\omega, x_{1}\right)\right\| \\
< & +a(\omega) c(\omega)\left[\left\|x_{1}-T\left(\omega, x_{2}\right)\right\|+\left\|x_{2}-T\left(\omega, x_{1}\right)\right\|\right] \\
& +b(\omega)\left\|x_{1}-T\left(\omega, x_{1}\right)\right\| .
\end{aligned}
$$

As $\varepsilon>0$ is arbitrary, it follows that

$$
\begin{aligned}
\left\|T\left(\omega, x_{1}\right)-T\left(\omega, x_{2}\right)\right\| \leq & a(\omega) c(\omega)\left[\left\|x_{1}-T\left(\omega, x_{2}\right)\right\|+\left\|x_{2}-T\left(\omega, x_{1}\right)\right\|\right] \\
& +b(\omega)\left\|x_{1}-T\left(\omega, x_{1}\right)\right\| .
\end{aligned}
$$

Case III:

Suppose

$$
\left\|T\left(\omega, s_{1}\right)-T\left(\omega, s_{2}\right)\right\| \leq a(\omega)\left\|s_{1}-s_{2}\right\|+b(\omega)\left\|s_{2}-T\left(\omega, s_{2}\right)\right\| .
$$

Now

$$
\begin{aligned}
\left\|T\left(\omega, x_{1}\right)-T\left(\omega, x_{2}\right)\right\| \leq & \left\|T\left(\omega, x_{1}\right)-T\left(\omega, s_{1}\right)\right\|+\left\|T\left(\omega, s_{1}\right)-T\left(\omega, s_{2}\right)\right\| \\
& +\left\|T\left(\omega, s_{2}\right)-T\left(\omega, x_{2}\right)\right\| \\
\leq & \left\|T\left(\omega, x_{1}\right)-T\left(\omega, s_{1}\right)\right\|+\left\|T\left(\omega, s_{2}\right)-T\left(\omega, x_{2}\right)\right\|+a(\omega)\left\|s_{1}-s_{2}\right\| \\
& +b(\omega)\left\|s_{2}-T\left(\omega, s_{2}\right)\right\| .
\end{aligned}
$$

Using (3.3), (3.6), (3.17), by a routine check-up, we get

$$
\begin{aligned}
\left\|T\left(\omega, x_{1}\right)-T\left(\omega, x_{2}\right)\right\| \leq & \left\|T\left(\omega, x_{1}\right)-T\left(\omega, s_{1}\right)\right\|+(1+b(\omega))\left\|T\left(\omega, s_{2}\right)-T\left(\omega, x_{2}\right)\right\| \\
& +a(\omega)\left\|s_{1}-x_{1}\right\|+(a(\omega)+b(\omega))\left\|x_{2}-s_{2}\right\| \\
& +a(\omega)\left\|x_{1}-x_{2}\right\|+b(\omega)\left\|x_{2}-T\left(\omega, x_{2}\right)\right\| .
\end{aligned}
$$

Since for a particular $\omega \in \Omega, T(\omega, x)$ is a continuous function of $x$, by using (3.9), (3.10), (3.11), (3.12) and for such a choice of $\delta_{1}, \delta_{2}$, we get from relation (3.18)

$$
\begin{aligned}
\left\|T\left(\omega, x_{1}\right)-T\left(\omega, x_{2}\right)\right\| & \leq \frac{\varepsilon}{8}(2+2 a(\omega)+2 b(\omega))+a(\omega)\left\|x_{1}-x_{2}\right\|+b(\omega)\left\|x_{2}-T\left(\omega, x_{2}\right)\right\| \\
& =\frac{\varepsilon}{2}+a(\omega)\left\|x_{1}-x_{2}\right\|+b(\omega)\left\|x_{2}-T\left(\omega, x_{2}\right)\right\| .
\end{aligned}
$$

As $\varepsilon>0$ is arbitrary, it follows that

$$
\left\|T\left(\omega, x_{1}\right)-T\left(\omega, x_{2}\right)\right\| \leq a(\omega)\left\|x_{1}-x_{2}\right\|+b(\omega)\left\|x_{2}-T\left(\omega, x_{2}\right)\right\| .
$$

Case IV: 


\section{Suppose}

$$
\begin{aligned}
\left\|T\left(\omega, s_{1}\right)-T\left(\omega, s_{2}\right)\right\| \leq & a(\omega) c(\omega)\left[\left\|s_{1}-T\left(\omega, s_{2}\right)\right\|+\left\|s_{2}-T\left(\omega, s_{1}\right)\right\|\right] \\
& +b(\omega)\left\|s_{2}-T\left(\omega, s_{2}\right)\right\| .
\end{aligned}
$$

Now

$$
\begin{aligned}
\left\|T\left(\omega, x_{1}\right)-T\left(\omega, x_{2}\right)\right\| \leq & \left\|T\left(\omega, x_{1}\right)-T\left(\omega, s_{1}\right)\right\|+\left\|T\left(\omega, s_{1}\right)-T\left(\omega, s_{2}\right)\right\| \\
& +\left\|T\left(\omega, s_{2}\right)-T\left(\omega, x_{2}\right)\right\| \\
\leq & \left\|T\left(\omega, x_{1}\right)-T\left(\omega, s_{1}\right)\right\|+\left\|T\left(\omega, s_{2}\right)-T\left(\omega, x_{2}\right)\right\| \\
& +a(\omega) c(\omega)\left[\left\|s_{1}-T\left(\omega, s_{2}\right)\right\|+\left\|s_{2}-T\left(\omega, s_{1}\right)\right\|\right] \\
& +b(\omega)\left\|s_{2}-T\left(\omega, s_{2}\right)\right\| .
\end{aligned}
$$

Using (3.4), (3.5), (3.6) and (3.20), we get by a routine check-up

$$
\begin{aligned}
\left\|T\left(\omega, x_{1}\right)-T\left(\omega, x_{2}\right)\right\| \leq & (1+a(\omega) c(\omega))\left\|T\left(\omega, x_{1}\right)-T\left(\omega, s_{1}\right)\right\| \\
& +(1+a(\omega) c(\omega)+b(\omega))\left\|T\left(\omega, x_{2}\right)-T\left(\omega, s_{2}\right)\right\| \\
& +a(\omega) c(\omega)\left\|s_{1}-x_{1}\right\|+(a(\omega) c(\omega)+b(\omega))\left\|s_{2}-x_{2}\right\| \\
& +a(\omega) c(\omega)\left[\left\|x_{1}-T\left(\omega, x_{2}\right)\right\|+\left\|x_{2}-T\left(\omega, x_{1}\right)\right\|\right] \\
& +b(\omega)\left\|x_{2}-T\left(\omega, x_{2}\right)\right\| .
\end{aligned}
$$

Since for a particular $\omega \in \Omega, T(\omega, x)$ is a continuous function of $x$, by using (3.9), (3.10), (3.11), (3.12) and for such a choice of $\delta_{1}, \delta_{2}$, we get by (3.21)

$$
\begin{aligned}
\left\|T\left(\omega, x_{1}\right)-T\left(\omega, x_{2}\right)\right\| \leq & (1+a(\omega) c(\omega)) \frac{\varepsilon}{8}+(1+a(\omega) c(\omega)+b(\omega)) \frac{\varepsilon}{8} \\
& +a(\omega) c(\omega) \frac{\varepsilon}{8}+(a(\omega) c(\omega)+b(\omega)) \frac{\varepsilon}{8} \\
& +a(\omega) c(\omega)\left[\left\|x_{1}-T\left(\omega, x_{2}\right)\right\|+\left\|x_{2}-T\left(\omega, x_{1}\right)\right\|\right] \\
& +b(\omega)\left\|x_{2}-T\left(\omega, x_{2}\right)\right\| \\
< & \frac{24-4 a(\omega)}{8-a(\omega)} \frac{\varepsilon}{4}+a(\omega) c(\omega)\left[\left\|x_{1}-T\left(\omega, x_{2}\right)\right\|+\left\|x_{2}-T\left(\omega, x_{1}\right)\right\|\right] \\
& +b(\omega)\left\|x_{2}-T\left(\omega, x_{2}\right)\right\| \\
< & \varepsilon+a(\omega) c(\omega)\left[\left\|x_{1}-T\left(\omega, x_{2}\right)\right\|+\left\|x_{2}-T\left(\omega, x_{1}\right)\right\|\right] \\
& +b(\omega)\left\|x_{2}-T\left(\omega, x_{2}\right)\right\| .
\end{aligned}
$$

As $\varepsilon>0$ is arbitrary, it follows that

$$
\begin{aligned}
\left\|T\left(\omega, x_{1}\right)-T\left(\omega, x_{2}\right)\right\| \leq & a(\omega) c(\omega)\left[\left\|x_{1}-T\left(\omega, x_{2}\right)\right\|+\left\|x_{2}-T\left(\omega, x_{1}\right)\right\|\right] \\
& +b(\omega)\left\|x_{2}-T\left(\omega, x_{2}\right)\right\| .
\end{aligned}
$$


Combining (3.13), (3.16), (3.19) and (3.22), we get

$$
\begin{aligned}
\left\|T\left(\omega, x_{1}\right)-T\left(\omega, x_{2}\right)\right\| \leq & a(\omega) \max \left\{\left\|x_{1}-x_{2}\right\|, c(\omega)\left[\left\|x_{1}-T\left(\omega, x_{2}\right)\right\|+\left\|x_{2}-T\left(\omega, x_{1}\right)\right\|\right]\right\} \\
& +b(\omega) \max \left\{\left\|x_{1}-T\left(\omega, x_{1}\right)\right\|,\left\|x_{2}-T\left(\omega, x_{2}\right)\right\|\right\} .
\end{aligned}
$$

Thus, $\omega \in \bigcap_{x_{1}, x_{2} \in X} C_{x_{1}, x_{2}} \cap A \cap B$, which implies

$$
\bigcap_{s_{1}, s_{2} \in S} C_{s_{1}, s_{2}} \cap A \cap B \subset \bigcap_{x_{1}, x_{2} \in X} C_{x_{1}, x_{2}} \cap A \cap B
$$

Also,

$$
\bigcap_{x_{1}, x_{2} \in X} C_{x_{1}, x_{2}} \cap A \cap B \subset \bigcap_{s_{1}, s_{2} \in S} C_{s_{1}, s_{2}} \cap A \cap B
$$

Thus,

$$
\bigcap_{x_{1}, x_{2} \in X} C_{x_{1}, x_{2}} \cap A \cap B=\bigcap_{s_{1}, s_{2} \in S} C_{s_{1}, s_{2}} \cap A \cap B
$$

Let $N^{\prime}=\bigcap_{s_{1}, s_{2} \in S} C_{s_{1}, s_{2}} \cap A \cap B$, then $\mu\left(N^{\prime}\right)=1$.

So, for each $\omega \in N^{\prime}, T(\omega, x)$ is a deterministic operator due to Ćirić [40]. Hence, $T$ has a unique fixed point in $X$.

Theorem 3.2 Let $X$ be a separable Banach space and $(\Omega, \beta, \mu)$ be a complete probability measure space. Let $T: \Omega \times X \rightarrow X$ be a continuous random operator such that for $\omega \in \Omega$, $T$ satisfies

$$
\begin{aligned}
\left\|T\left(\omega, x_{1}\right)-T\left(\omega, x_{2}\right)\right\| \leq & a(\omega)\left\|x_{1}-x_{2}\right\|+b(\omega) \max \left\{\left\|x_{1}-T\left(\omega, x_{1}\right)\right\|,\left\|x_{2}-T\left(\omega, x_{2}\right)\right\|\right\} \\
& +c(\omega)\left[\left\|x_{1}-T\left(\omega, x_{2}\right)\right\|+\left\|x_{2}-T\left(\omega, x_{2}\right)\right\|\right]
\end{aligned}
$$

for all $x, y \in X$, where $a(\omega), b(\omega), c(\omega)$ are real-valued random variables such that

$$
0<a(\omega)<1, \quad b(\omega) \geq 0, \quad c(\omega) \geq 0 \quad \text { satisfying } \quad a(\omega)+c(\omega)>0
$$

and

$$
a(\omega)+b(\omega)+\frac{7}{3} c(\omega)=1 \quad \text { almost surely, }
$$

then $T$ has a unique random fixed point in $X$.

Proof Set $a(\omega)+\frac{7}{3} c(\omega)=a_{1}(\omega)$.

Then $a_{1}(\omega)+b(\omega)=1$ and we have

$$
\begin{gathered}
a(\omega)\left\|x_{1}-x_{2}\right\|+b(\omega) \max \left\{\left\|x_{1}-T\left(\omega, x_{1}\right)\right\|,\left\|x_{2}-T\left(\omega, x_{2}\right)\right\|\right\} \\
+c(\omega) \cdot \frac{7}{3} \cdot \frac{3}{7}\left[\left\|x_{1}-T\left(\omega, x_{2}\right)\right\|+\left\|x_{2}-T\left(\omega, x_{1}\right)\right\|\right]
\end{gathered}
$$




$$
\begin{aligned}
\leq & \left(a(\omega)+\frac{7}{3} c(\omega)\right) \max \left\{\left\|x_{1}-x_{2}\right\|, \frac{3}{7}\left[\left\|x_{1}-T\left(\omega, x_{2}\right)\right\|+\left\|x_{2}-T\left(\omega, x_{1}\right)\right\|\right]\right\} \\
& +b(\omega) \max \left\{\left\|x_{1}-T\left(\omega, x_{1}\right)\right\|,\left\|x_{2}-T\left(\omega, x_{2}\right)\right\|\right\} .
\end{aligned}
$$

Since $\frac{3}{7}<\frac{4-a(\omega)}{8-a(\omega)}$, the relation (3.23), (3.24) and (3.25) would imply Theorem 3.1 with $a_{1}(\omega)+$ $b(\omega)=1$. Therefore, we can apply Theorem 3.1 and consequently $T$ has a unique random fixed point in $X$.

We now give a couple of examples in support of Theorem 3.1 and Theorem 3.2.

Example 3.3 Let $E=[-2,5] \subset \mathbb{R}$ with the usual norm of reals.

Consider $\Omega=[-2,5]$ and let $\beta$ be a $\sigma$-algebra of Lebesgue measurable sets of $[-2,5]$.

Define $T: \Omega \times E \rightarrow E$ by $T(\omega, x)=\frac{x}{7}$, where $x \in E$ and $\omega \in \Omega$.

By a routine check-up, we see that the condition of Theorem 3.1 is satisfied whenever $a(\omega)=\frac{1}{7}, b(\omega)=\frac{6}{7}$ and $0 \leq c(\omega) \leq \frac{27}{55}$. The function $x: \Omega \rightarrow E$ with $x(\omega)=0$ is a unique random fixed point of $T$.

By considering $E$ and $\Omega$ as above, we take $a(\omega)=\frac{1}{7}, b(\omega)=\frac{6}{7}$ and $c(\omega)=0$. We see that condition (3.23) of Theorem 3.2 is satisfied and the function $x: \Omega \rightarrow E$ with $x(\omega)=0$ is the unique random fixed point of $T$.

Example 3.4 Let $X=\mathbb{R}$ with the usual norm of reals. Let $\Omega=\mathbb{R}$. $\beta$ be a $\sigma$-algebra of Lebesgue measurable sets of $\mathbb{R}$.

Define $T: \Omega \times X \rightarrow X$ by $T(\omega, x)=\frac{1}{3}$.

All the conditions of Theorem 3.1 and Theorem 3.2 are satisfied. In both of the cases, we see that the function $x: \Omega \rightarrow E$ with $x(\omega)=\frac{1}{3}$ is the unique random fixed point of $T$.

\section{Application to a random nonlinear integral equation}

Here we apply Theorem 3.1 to prove the existence of a solution in a Banach space of a random nonlinear integral equation of the following form:

$$
x(t ; \omega)=h(t ; \omega)+\int_{S} k(t, s ; \omega) f(s, x(s ; \omega)) d \mu_{0}(s),
$$

where

(i) $S$ is a locally compact metric space with a metric $d$ on $S \times S$ equipped with a complete $\sigma$-finite measure $\mu_{0}$ defined on the collection of Borel subsets of $S$;

(ii) $\omega \in \Omega$, where $\omega$ is the supporting element of a set of probability measure space $(\Omega, \beta, \mu)$;

(iii) $x(t ; \omega)$ is the unknown vector-valued random variable for each $t \in S$;

(iv) $h(t ; \omega)$ is the stochastic free term defined for $t \in S$;

(v) $k(t, s ; \omega)$ is the stochastic kernel defined for $t$ and $s$ in $S$ and

(vi) $f(t, x)$ is a vector-valued function of $t \in S$ and $x$.

The integral in equation (4.1) is interpreted as a Bochner integral [43].

We shall further assume that $S$ is the union of a decreasing sequence of countable family of compact sets $\left\{C_{n}\right\}$ such that for any other compact set in $S$ there is a $C_{i}$ which contains it (see [44]). 
Definition 4.1 We define the space $C\left(S, L_{2}(\Omega, \beta, \mu)\right)$ to be the space of all continuous functions from $S$ into $L_{2}(\Omega, \beta, \mu)$ with the topology of uniform convergence on compact sets of $S$, that is, for each fixed $t \in S, x(t ; \omega)$ is a vector-valued random variable such that

$$
\|x(t ; \omega)\|_{L_{2}(\Omega, \beta, \mu)}^{2}=\int_{\Omega}|x(t ; \omega)|^{2} d \mu(\omega)<\infty
$$

It may be noted that $C\left(S, L_{2}(\Omega, \beta, \mu)\right)$ is a locally convex space (see [43]) whose topology is defined by a countable family of semi-norms given by

$$
\|x(t ; \omega)\|_{n}=\sup _{t \in C_{n}}\|x(t ; \omega)\|_{L_{2}(\Omega, \beta, \mu)}, \quad n=1,2, \ldots
$$

Moreover, $C\left(S, L_{2}(\Omega, \beta, \mu)\right)$ is complete relative to this topology since $L_{2}(\Omega, \beta, \mu)$ is complete.

We further define $B C=B C\left(S, L_{2}(\Omega, \beta, \mu)\right)$ to be the Banach space of all bounded continuous functions from $S$ into $L_{2}(\Omega, \beta, \mu)$ with the norm

$$
\|x(t ; \omega)\|_{B C}=\sup _{t \in S}\|x(t ; \omega)\|_{L_{2}(\Omega, \beta, \mu)} .
$$

Here the space $B C \subset C$ is the space of all second-order vector-valued functions defined on $S$ which are bounded and continuous in mean square. We will consider the function $h(t ; \omega)$ and $f(t, x(t ; \omega))$ to be in the space $C\left(S, L_{2}(\Omega, \beta, \mu)\right)$ with respect to the stochastic kernel. We assume that for each pair $(t, s), k(t, s ; \omega) \in L_{\infty}(\Omega, \beta, \mu)$ and denote the norm by

$$
\begin{aligned}
\|k(t, s ; \omega)\| & =\|k(t, s ; \omega)\|_{L_{\infty}(\Omega, \beta, \mu)} \\
& =\mu-\operatorname{ess} \sup _{\omega \in \Omega}|k(t, s ; \omega)| .
\end{aligned}
$$

Let us suppose that $k(t, s ; \omega)$ is such that $\|k(t, s ; \omega)\| \cdot\|x(s ; \omega)\|_{L_{2}(\Omega, \beta, \mu)}$ is $\mu_{0}$-integrable with respect to $s$ for each $t \in S$ and $x(s ; \omega)$ in $C\left(S, L_{2}(\Omega, \beta, \mu)\right)$, and let there exist a real-valued function $G$ defined $\mu_{0}$-a.e. on $S$, so that $G(S)\|x(s ; \omega)\|_{L_{2}(\Omega, \beta, \mu)}$ is $\mu_{0}$-integrable so that for each pair $(t, s) \in S \times S$,

$$
\|k(t, u ; \omega)-k(s, u ; \omega)\| \cdot\|x(u, \omega)\|_{L_{2}(\Omega, \beta, \mu)} \leq G(u)\|x(u, \omega)\|_{L_{2}(\Omega, \beta, \mu)},
$$

$\mu_{0}$-a.e. Further, for almost all $s \in S, k(t, s ; \omega)$ will be continuous in $t$ from $S$ into $L_{\infty}(\Omega, \beta, \mu)$.

We now define the random integral operator $T$ on $C\left(S, L_{2}(\Omega, \beta, \mu)\right)$ by

$$
(T x)(t ; \omega)=\int_{S} k(t, s ; \omega) x(s ; \omega) d \mu_{0}(s)
$$

where the integral is a Bochner integral. Moreover, we have that for each $t \in S,(T x)(t ; \omega) \in$ $L_{2}(\Omega, \beta, \mu)$ and that $(T x)(t ; \omega)$ is continuous in mean square by the Lebesgue dominated convergence theorem. So, $(T x)(t ; \omega) \in C\left(S, L_{2}(\Omega, \beta, \mu)\right)$. 
Definition 4.2 (see [29] and [45]) Let $B$ and $D$ be two Banach spaces. The pair $(B, D)$ is said to be admissible with respect to a random operator $T(\omega)$ if $T(\omega)(B) \subset D$.

Lemma 4.3 (see [28]) The linear operator $T$ defined by (4.2) is continuous from $C\left(S, L_{2}(\Omega\right.$, $\beta, \mu))$ into itself.

Lemma 4.4 (see [45] and [28]) If $T$ is a continuous linear operator from $C\left(S, L_{2}(\Omega, \beta, \mu)\right)$ into itself and $B, D \subset C\left(S, L_{2}(\Omega, \beta, \mu)\right)$ are Banach spaces stronger than $C\left(S, L_{2}(\Omega, \beta, \mu)\right)$ such that $(B, D)$ is admissible with respect to $T$, then $T$ is continuous from $B$ into $D$.

Remark 4.5 (see [28]) The operator $T$ defined by (4.2) is a bounded linear operator from $B$ into $D$.

A random solution of equation (4.1) will mean a function $x(t ; \omega)$ in $C\left(S, L_{2}(\Omega, \beta, \mu)\right)$ which satisfies equation (4.1) $\mu$-a.e.

We are now in a position to prove the following theorem.

Theorem 4.6 We consider the stochastic integral equation (4.1) subject to the following conditions:

(a) $B$ and $D$ are Banach spaces stronger than $C\left(S, L_{2}(\Omega, \beta, \mu)\right)$ such that $(B, D)$ is admissible with respect to the integral operator defined by (4.2);

(b) $x(t ; \omega) \rightarrow f(t, x(t ; \omega))$ is an operator from the set $Q(\rho)=\{x(t ; \omega): x(t ; \omega) \in D$, $\left.\|x(t ; \omega)\|_{D} \leq \rho\right\}$ into the space B satisfying

$$
\begin{aligned}
& \left\|f\left(t, x_{1}(t ; \omega)\right)-f\left(t, x_{2}(t ; \omega)\right)\right\|_{B} \\
& \leq a(\omega) \max \left\{\left\|x_{1}(t ; \omega)-x_{2}(t ; \omega)\right\|_{D}\right. \\
& \left.\quad c(\omega)\left[\left\|x_{1}(t ; \omega)-f\left(t, x_{2}(t ; \omega)\right)\right\|_{D}+\left\|x_{2}(t ; \omega)-f\left(t, x_{1}(t ; \omega)\right)\right\|_{D}\right]\right\} \\
& \quad+b(\omega) \max \left\{\left\|x_{1}(t ; \omega)-f\left(t, x_{1}(t ; \omega)\right)\right\|_{D}+\left\|x_{2}(t ; \omega)-f\left(t, x_{2}(t ; \omega)\right)\right\|_{D}\right\}
\end{aligned}
$$

for $x_{1}(t ; \omega), x_{2}(t ; \omega) \in Q(\rho), a(\omega), b(\omega), c(\omega)$ are real-valued random variables where $0<$ $a(\omega)<1$ and $a(\omega)+b(\omega)=1$ with $c(\omega) \leq \frac{4-a(\omega)}{8-a(\omega)}$ almost surely, and

(c) $h(t ; \omega) \in D$.

Then there exists a unique random solution of $(4.1)$ in $Q(\rho)$, provided $\frac{l(\omega)}{1-a(\omega) c(\omega)-b(\omega)}<1$ and

$$
\begin{aligned}
& \|h(t ; \omega)\|_{D}+l(\omega)\|f(t, 0)\|_{B}\left[\frac{1}{1-b(\omega)}+(1+b(\omega))\right. \\
& \left.\quad+\frac{1+a(\omega) c(\omega)}{1-a(\omega) c(\omega)-b(\omega)}+\frac{1+b(\omega)+a(\omega) c(\omega)}{1-a(\omega) c(\omega)}\right] \\
& \leq \rho\left(1-\frac{l(\omega)}{1-a(\omega) c(\omega)}\right), \text { where } l(\omega) \text { is the norm of } T(\omega) .
\end{aligned}
$$

Proof Define the operator $U(\omega)$ from $Q(\rho)$ into $D$ by

$$
(U x)(t ; \omega)=h(t ; \omega)+\int_{S} k(t, s ; \omega) f(s, x(s ; \omega)) d \mu_{0}(s) .
$$


Now

$$
\begin{aligned}
\|(U x)(t ; \omega)\|_{D} & \leq\|h(t ; \omega)\|_{D}+l(\omega)\|f(t, x(t ; \omega))\|_{B} \\
& \leq\|h(t ; \omega)\|_{D}+l(\omega)\|f(t ; 0)\|_{B}+l(\omega)\|f(t, x(t ; \omega))-f(t ; 0)\|_{B} .
\end{aligned}
$$

Then from (4.3) of this theorem,

$$
\begin{aligned}
& \|f(t, x(t ; \omega))-f(t, 0)\|_{B} \\
& \leq a(\omega) \max \left\{\|x(t ; \omega)\|_{D}, c(\omega)\left[\|x(t ; \omega)-f(t ; 0)\|_{D}+\|f(t, x(t ; \omega))\|_{D}\right]\right\} \\
& \quad+b(\omega) \max \left\{\|x(t ; \omega)-f(t, x(t ; \omega))\|_{D},\|f(t ; 0)\|_{D}\right\} .
\end{aligned}
$$

Suppose

$$
\begin{aligned}
\|f(t, x(t ; \omega))-f(t ; 0)\|_{B} \leq & a(\omega)\|x(t ; \omega)\|_{D}+b(\omega)\|x(t ; \omega)-f(t, x(t ; \omega))\|_{D} \\
\leq & a(\omega)\|x(t ; \omega)\|_{D}+b(\omega)\|x(t ; \omega)\|_{D} \\
& +b(\omega)\|f(t, x(t ; \omega))-f(t ; 0)\|_{D}+b(\omega)\|f(t ; 0)\|_{D} .
\end{aligned}
$$

Hence,

$$
\|f(t, x(t ; \omega))-f(t ; 0)\|_{B} \leq \frac{a(\omega)+b(\omega)}{1-b(\omega)} \rho+\frac{b(\omega)}{1-b(\omega)}\|f(t ; 0)\|_{B} .
$$

So, by (4.4)

$$
\begin{aligned}
\left\|\left(U_{x}\right)(t ; x)\right\|_{D} \leq & \|h(t ; \omega)\|_{D}+l(\omega)\|f(t ; 0)\|_{B} \\
& +\frac{a(\omega)+b(\omega)}{1-b(\omega)} l(\omega) \rho+\frac{b(\omega) l(\omega)}{1-b(\omega)}\|f(t ; 0)\|_{B} \\
< & \|h(t ; \omega)\|_{D}+\frac{1}{1-b(\omega)} l(\omega)\|f(t ; 0)\|_{B}+\frac{1}{1-b(\omega)} l(\omega) \rho \\
< & \rho .
\end{aligned}
$$

Again, suppose

$$
\|f(t, x(t ; \omega))-f(t ; 0)\|_{B} \leq a(\omega)\|x(t ; \omega)\|_{D}+b(\omega)\|f(t ; 0)\|_{D} .
$$

So,

$$
\|f(t, x(t ; \omega))-f(t ; 0)\|_{B} \leq a(\omega) \rho+b(\omega)\|f(t ; 0)\|_{B} .
$$

Therefore, by (4.6)

$$
\begin{aligned}
\left\|\left(U_{x}\right)(t ; \omega)\right\|_{D} & \leq\|h(t ; \omega)\|_{D}+l(\omega)\|f(t ; 0)\|_{B}+l(\omega) a(\omega) \rho+l(\omega) b(\omega)\|f(t ; 0)\|_{B} \\
& =\|h(t ; \omega)\|_{D}+(1+b(\omega)) l(\omega)\|f(t ; 0)\|_{B}+l(\omega) a(\omega) \rho \\
& <\rho .
\end{aligned}
$$


Also, suppose

$$
\begin{aligned}
\|f(t, x(t ; \omega))-f(t ; 0)\|_{B} \leq & a(\omega) c(\omega)\left[\|x(t ; \omega)-f(t ; 0)\|_{D}+\|f(t, x(t ; \omega))\|_{D}\right] \\
& +b(\omega)\|x(t ; \omega)-f(t, x(t ; \omega))\|_{D} \\
\leq & a(\omega) c(\omega)\|x(t ; \omega)\|_{D}+a(\omega) c(\omega)\|f(t ; 0)\|_{D} \\
& +a(\omega) c(\omega)\|f(t ; x(t ; \omega))-f(t ; 0)\|_{D} \\
& +a(\omega) c(\omega)\|f(t ; 0)\|_{D}+b(\omega)\|x(t ; \omega)\|_{D} \\
& +b(\omega)\|f(t, x(t ; \omega))-f(t ; 0)\|_{D}+b(\omega)\|f(t ; 0)\|_{D} .
\end{aligned}
$$

Then

$$
\begin{aligned}
\|f(t, x(t ; \omega))-f(t ; 0)\|_{B} \leq & \frac{a(\omega) c(\omega)+b(\omega)}{1-a(\omega) c(\omega)-b(\omega)} \rho \\
& +\left(\frac{2 a(\omega) c(\omega)+b(\omega)}{1-a(\omega) c(\omega)-b(\omega)}\right)\|f(t ; 0)\|_{B} .
\end{aligned}
$$

Therefore, by (4.8)

$$
\begin{aligned}
\|(U x)(t ; \omega)\|_{D} \leq & \|h(t ; \omega)\|_{D}+l(\omega)\|f(t ; 0)\|_{B} \\
& +l(\omega) \frac{a(\omega) c(\omega)+b(\omega)}{1-a(\omega) c(\omega)-b(\omega)} \rho \\
& +\frac{2 a(\omega) c(\omega)+b(\omega)}{1-a(\omega) c(\omega)-b(\omega)} l(\omega)\|f(t ; 0)\|_{B} \\
= & \|h(t ; \omega)\|_{D}+\frac{1+a(\omega) c(\omega)}{1-a(\omega) c(\omega)-b(\omega)} l(\omega)\|f(t ; 0)\|_{B} \\
& +l(\omega) \frac{a(\omega) c(\omega)+b(\omega)}{1-a(\omega) c(\omega)-b(\omega)} \rho \\
< & \rho .
\end{aligned}
$$

Suppose

$$
\begin{aligned}
\|f(t, x(t ; \omega))-f(t ; 0)\|_{B} \leq & a(\omega) c(\omega)\left[\|x(t ; \omega)-f(t ; 0)\|_{D}+\|f(t, x(t ; \omega))\|_{D}\right] \\
& +b(\omega)\|f(t ; 0)\|_{D} \\
\leq & a(\omega) c(\omega)\|x(t ; \omega)\|_{D}+a(\omega) c(\omega)\|f(t ; 0)\|_{D} \\
& +a(\omega) c(\omega)\|f(t, x(t ; \omega))-f(t ; 0)\|_{D} \\
& +a(\omega) c(\omega)\|f(t ; 0)\|_{D}+b(\omega)\|f(t ; 0)\|_{D^{.}}
\end{aligned}
$$

So,

$$
\|f(t, x(t ; \omega))-f(t ; 0)\|_{B} \leq \frac{a(\omega) c(\omega)}{1-a(\omega) c(\omega)} \rho+\frac{b(\omega)+2 a(\omega) c(\omega)}{1-a(\omega) c(\omega)}\|f(t ; 0)\|_{B} .
$$


Therefore, by (4.10)

$$
\begin{aligned}
\|(U x)(t ; \omega)\|_{D} \leq & \|h(t ; \omega)\|_{D}+l(\omega)\|f(t ; 0)\|_{B}+\frac{a(\omega) c(\omega)}{1-a(\omega) c(\omega)} l(\omega) \rho \\
& +\frac{b(\omega)+2 a(\omega) c(\omega)}{1-a(\omega) c(\omega)} l(\omega)\|f(t ; 0)\|_{B} \\
= & \|h(t ; \omega)\|_{D}+\frac{a(\omega) c(\omega)}{1-a(\omega) c(\omega)} l(\omega) \rho+\frac{1+b(\omega)+a(\omega) c(\omega)}{1-a(\omega) c(\omega)} l(\omega)\|f(t ; 0)\|_{B} \\
< & \rho .
\end{aligned}
$$

Then by (4.5), (4.7), (4.9) and (4.11), we get $(U x)(t ; \omega) \in Q(\rho)$.

Then for $x_{1}(t ; \omega), x_{2}(t ; \omega) \in Q(\rho)$. We have by condition (b)

$$
\begin{aligned}
&\left\|\left(U x_{1}\right)(t ; \omega)-\left(U x_{2}\right)(t ; \omega)\right\|_{D} \\
&=\left\|\int_{S} k(t, s ; \omega)\left[f\left(s, x_{1}(s ; \omega)\right)-f\left(s, x_{2}(s ; \omega)\right)\right] d \mu_{0}(s)\right\|_{D} \\
& \leq l(\omega)\left\|f\left(t, x_{1}(t ; \omega)\right)-f\left(t, x_{2}(t ; \omega)\right)\right\|_{B} \\
& \leq a(\omega) \max \left\{\left\|x_{1}(t ; \omega)-x_{2}(t ; \omega)\right\|,\right. \\
&\left.c(\omega)\left[\left\|x_{1}(t ; \omega)-\left(U x_{2}\right)(t ; \omega)\right\|_{D}+\left\|x_{2}(t ; \omega)-\left(U x_{1}\right)(t ; \omega)\right\|_{D}\right]\right\} \\
&+b(\omega) \max \left\{\left\|x_{1}(t ; \omega)-\left(U x_{1}\right)(t ; \omega)\right\|_{D},\left\|x_{2}(t ; \omega)-\left(U x_{2}\right)(t ; \omega)\right\|_{D}\right\} .
\end{aligned}
$$

Since $\frac{l(\omega)}{1-a(\omega) c(\omega)-b(\omega)}<1$.

Therefore, $U(\omega)$ is a random contractive nonlinear operator on $Q(\rho)$. Hence, by Theorem 3.1, there exists a random fixed point of $U(\omega)$, which is the random solution of equation (4.1).

Example 4.7 Consider the following nonlinear stochastic integral equation:

$$
x(t ; \omega)=\int_{0}^{\infty} \frac{e^{-t-s}}{8(1+|x(s ; \omega)|)} d s .
$$

Comparing with (4.1), we see that

$$
h(t ; \omega)=0, \quad k(t, s ; \omega)=\frac{1}{4} e^{-t-s}, \quad f(s, x(s ; \omega))=\frac{1}{2(1+|x(s ; \omega)|)} .
$$

By routine calculation, it is easy to show that (4.3) is satisfied with $a(\omega)=\frac{1}{2}, b(\omega)=\frac{1}{2}$ and $0 \leq c(\omega) \leq \frac{7}{15}$.

Comparing with integral operator equation (4.2), we see that the norm of $T(\omega)$ is $l(\omega)=\frac{1}{4}$.

Also, we see that $\frac{l(\omega)}{1-a(\omega) c(\omega)-b(\omega)}<1$. So, all the conditions of Theorem 4.6 are satisfied and hence there exists a random fixed point of the integral operator $T$ satisfying (4.2). 


\section{Authors' contributions}

Both the authors are jointly responsible for the present research work and have equally contributed to the research work. Both the authors have read and approved the final manuscript.

\section{Author details}

'Department of Mathematics, The University of Burdwan, Burdwan, West Bengal 713104, India. ${ }^{2}$ Burdwan Railway Balika Vidyapith High School, Khalasipara, Burdwan, West Bengal 713101, India.

\section{Acknowledgements}

Authors remain grateful to the honorable reviewers for their kind suggestions for improvement of our paper.

Received: 25 June 2012 Accepted: 6 November 2012 Published: 22 November 2012

\section{References}

1. Bharucha-Reid, AT: Random Integral Equations. Academic Press, New York (1972)

2. Debnath, L, Mikusinski, P: Introduction to Hilbert Spaces with Application. Academic Press, Boston (2005)

3. Joshi, MC, Bose, RK: Some Topics in Nonlinear Functional Analysis. Wiley, New York (1984)

4. Špaček, A: Zufällige Gleichungen. Czechoslov. Math. J. 5(80), 462-466 (1955)

5. Hanš, O: Random operator equations. In: Proceedings of 4th Berkeley Sympos. Math. Statist. Prob., vol. II, part I, pp. 185-202. University of California Press, Berkeley (1961)

6. Hanš, O: Reduzierende zufällige transformationen. Czechoslov. Math. J. 7(82), 154-158 (1957)

7. Bharucha-Reid, AT: Fixed point theorems in probabilistic analysis. Bull. Am. Math. Soc. 82(5), 641-657 (1976)

8. Itoh, S: Random fixed-point theorems with an application to random differential equations in Banach spaces. J. Math. Anal. Appl. 67(2), 261-273 (1979)

9. Mukherjee, A: Transformation aleatoires separable theorem all point fixed aleatoire. C. R. Acad. Sci. Paris, Ser. A-B 263, 393-395 (1966)

10. Rothe, E: Zur theorie der topologischen ordnung und der Vektorfelder in Banachschen Räumen. Compos. Math. 5, 177-197 (1938)

11. Sehgal, VM, Waters, C: Some random fixed point theorems for condensing operators. Proc. Am. Math. Soc. 90(1), 425-429 (1984)

12. Kumam, P, Kumam, W: Random fixed points of multivalued random operators with property (D). Random Oper Stoch. Equ. 15(2), 127-136 (2007)

13. Kumam, $\mathrm{P}$, Plubtieng, S: Random coincidence and random common fixed points of nonlinear multivalued random operators. Thai J. Math. 5(3), 155-163 (2007) (Special issue)

14. Kumam, P, Plubtieng, S: Random common fixed point theorems for a pair of multi-valued and single-valued nonexpansive random operators in a separable Banach space. Indian J. Math. 51(1), 101-115 (2009)

15. Kumam, P, Plubtieng, S: Random fixed point theorem for multivalued nonexpansive operators in uniformly nonsquare Banach spaces. Random Oper. Stoch. Equ. 14(1), 35-44 (2006)

16. Kumam, P, Plubtieng, S: Random fixed point theorems for asymptotically regular random operators. Demonstr. Math. XLII(1), 131-141 (2009)

17. Kumam, P, Plubtieng, S: Random fixed point theorems for multivalued nonexpansive non-self random operators. J. Appl. Math. Stoch. Anal. 2006, Article ID 43796 (2006)

18. Kumam, P, Plubtieng, S: Some random fixed point theorem for set-valued nonexpansive non-self operator. In: Takahashi, W, Tanaka, T (eds.) Nonlinear Analysis and Convex Analysis. Proceedings of the 4th International Conference (NACA 2005), Okinawa, Japan, June 30-July 4, 2005, pp. 287-295. Yokohama Publishers, Yokohama (2005)

19. Kumam, P, Plubtieng, S: Some random fixed point theorems for non-self nonexpansive random operators. Turk. J. Math. 30, 359-372 (2006)

20. Kumam, P, Plubtieng, S: The characteristic of noncompact convexity and random fixed point theorem for set-valued operators. Czechoslov. Math. J. 57(132), 269-279 (2007)

21. Kumam, P, Plubtieng, S: Viscosity approximation methods of random fixed point solutions and random variational inequalities in Hilbert spaces. Asian-Eur. J. Math. 70(1), 81-107 (2011)

22. Kumam, P: Fixed point theorem and random fixed point theorems for set-valued non-self-mappings. Thai J. Math. 2(2), 295-307 (2004)

23. Kumam, P: Random common fixed points of single-valued and multivalued random operators in a uniformly convex Banach space. J. Comput. Anal. Appl. 13(2), 368-375 (2011)

24. Kumam, W, Kumam, P: Random fixed point theorems for multivalued subsequentially limit-contractive maps satisfying inwardness conditions. J. Comput. Anal. Appl. 14(2), 239-251 (2012)

25. Sintunavarat, W, Kumam, P, Patthanangkoor, P: Common random fixed points for multivalued random operators without $S$ and $T$-weakly commuting random operators. Random Oper. Stoch. Equ. 17(4), 381-388 (2009)

26. Saha, M: On some random fixed point of mappings over a Banach space with a probability measure. Proc. Natl. Acad. Sci. India, Sect. A 76, 219-224 (2006)

27. Saha, M, Debnath, L: Random fixed point of mappings over a Hilbert space with a probability measure. Adv. Stud. Contemp. Math. 1, 79-84 (2007)

28. Padgett, WJ: On a nonlinear stochastic integral equation of the Hammerstein type. Proc. Am. Math. Soc. 38, 625-631 (1973)

29. Achari, J: On a pair of random generalized nonlinear contractions. Int. J. Math. Math. Sci. 6(3), 467-475 (1983)

30. Saha, M, Dey, D: Some random fixed point theorems for $(\theta, L)$-weak contractions. Hacet. J. Math. Stat. (accepted and to appear)

31. Banach, S: Sur les opérations dans les ensembles abstraits et leur application aux équations intégrals. Fundam. Math. 3, 133-181 (1922)

32. Goebel, K, Kirk, WA: Topics in Metric Fixed Point Theory. Cambridge University Press, New York (1990)

33. Smart, OR: Fixed Point Theorems. Cambridge University Press, London (1974) 
34. Greguš, M: A fixed point theorem in Banach space. Boll. Unione Mat. Ital., A 5(7), 193-198 (1980)

35. Ćirić, L: A generalization of Banach's contraction principle. Proc. Am. Math. Soc. 45, 267-273 (1974)

36. Ćirić, L: On a common fixed point theorem of a Greguš type. Publ. Inst. Math. (Belgr.) 49(63), 174-178 (1991)

37. Diviccaro, ML, Fisher, B, Sessa, S: A common fixed point theorem of Greguš type. Publ. Math. (Debr.) 34, 83-89 (1987)

38. Fisher, B, Sessa, S: On a fixed point theorem of Greguš. Int. J. Math. Math. Sci. 9(1), 23-28 (1986)

39. Mukherjea, RN, Verma, V: A note on a fixed point theorem of Greguš. Math. Jpn. 33, 745-749 (1988)

40. Ćirić, L: On a generalization of a Greguš fixed point theorem. Czechoslov. Math. J. 50(3), 449-458 (2000)

41. Karapinar, E: Some nonunique fixed point theorems of Ćirić type on cone metric spaces. Abstr. Appl. Anal. 2010, Article ID 123094 (2010). doi:10.1155/2010/123094

42. Karapinar, E, Chi, KP, Thanh, TD: A generalization of Ćirić quasicontractions. Abstr. Appl. Anal. 2012, Article ID 518734 (2012). doi:10.1155/2012/518734

43. Yosida, K: Functional Analysis. Springer, Berlin (1965)

44. Arens, RF: A topology for spaces of transformations. Ann. Math. 47(2), 480-495 (1946)

45. Lee, ACH, Padgett, WJ: On random nonlinear contraction. Math. Syst. Theory 11, 77-84 (1977)

doi:10.1186/1687-1812-2012-209

Cite this article as: Saha and Ganguly: Random fixed point theorem on a Ćirić-type contractive mapping and its consequence. Fixed Point Theory and Applications 2012 2012:209.

\section{Submit your manuscript to a SpringerOpen ${ }^{\circ}$ journal and benefit from:}

- Convenient online submission

- Rigorous peer review

- Immediate publication on acceptance

- Open access: articles freely available online

- High visibility within the field

- Retaining the copyright to your article 